\title{
La création féminine comme récit d'une intimité
}

\author{
Michelle Coquillat
}

\section{Women's Creative Process as Narrative of Intimacy}

In her text, Michelle Coquillat suggests that theories of poetics work to deny women the power of creation. In spite of their substantial differences, the Biblical act of creation ex nihilo, the post-romantic notion of a solitary artist expressing his innermost being, and the modernist drive to "make it new," share an emphasis upon originality. Originality, of course, is a quality in which work by women is often found wanting. Women, whose creativity is understood historically in terms of maternity, in terms of procreation, are constructed as ontologically ineligible for the creative act. Insofar as a woman needs another in order to bring a being into the world, her act is considered irretrievably secondary, unoriginal. What is more, when childbirth is used as a metaphor for the process of creation, the artistic activity of women is thrown further into question.

Creating, Coquillat observes, requires of a woman artist a double effort: that she resist, indeed critique, what society teaches her about herself and the "derivative" quality of her work, and that she inquire within herself, that she explore her interiority. The latter exploration is relatively unproblematical for men; it may render a man a social outcast, but it does not threaten his legitimacy and visibility as an artist. For women, whose roles are conventionally limited to the sphere of the intimate, such an exploration is more difficult - but nevertheless promises a radical reconceptualization of the play of emotion and otherness, secondariness, in the work of creating.

Le thème de l'intimité, c'est-à-dire avant tout de l'intériorité, est, dans l'art et la poétique, la marque souveraine de la création masculine, telle qu'elle a pu s'afficher en particulier depuis la fin du 18e siècle avec Rousseau en France, ou Goethe par exemple en Allemagne. Car, avant toute chose, la création est un lieu de lutte et de rivalité où s'exprime, au plus haut point, la différence des sexes. Avec une constance parfaite, la théorisation poétique masculine s'est efforcée - et s'efforce encore -, d'exclure la femme de la puissance créatrice pour n'accorder qu'à 
l'homme la possibilité de l'effort nécessaire à la mise au monde symbolique de l'oeuvre. J'ai pu déterminer, dans mon travail sur la poétique du mâle, deux grands schémas créateurs dont le premier est analogique de l'acte de la Genèse et le second, sexualisé, va permettre de voir éclore le mythe du créateur solitaire, androgyne et profondément intime. Dans le premier schéma, celui qui est dominé par la création divine, tel Dieu, l'homme crée EXNIHILO, de rien, uneoeuvre profondément intime, qui est sa marque sur le monde. Empêtrée dans sa maternité, la femme, vouée à la procréation, est ontologiquement inapte, nous dit le créateur mâle, à cette création. En effet, elle a BESOIN de l'autre pour mettre au monde. La maternité est une dépendance, elle est le contraire de $l^{\prime} E X$ NIHILO, la femme ne peut créer de rien. (On s'imagine ce que la mise en évidence du clonage, c'est-à-dire une procréation sans spermatozoïde, peut apporter comme modification à cette vision, toujours actuelle par certains côtés, de la déficience créatrice féminine.) Ainsi, à cause de cette dépendance, même grande, la femme dans cette théorisation, est seconde. Il faut qu'on lui montre la voie. On pense à Chimène dans Le Cid, personnage héroïque mais non créateur, qui reconnaît devant Rodrigue qu'il lui a appris le chemin de la grandeur ( $T u$ n'as fait que le devoir d'un homme de bien/Mais aussi le faisant tu m'as appris le mien). Mise en place, une fois de plus, du rapport maître à disciple qui tient lieu d'héroïsme à la femme et la rejette dans la non-intimité, la non-intériorité, puisque c'est dans l'homme qu'elle prend son inspiration, alors que lui c'est en lui-même. En lui-même, puisque son acte est SANS MODÈLE, c'est un acte neuf.

Dès les prémices de la schématisation masculine de la création, on $s^{\prime}$ aperçoit que c'est parce qu' elle est mère que la femme doit être dépouillée de toute puissance symbolique. Par opposition, c'est EN LUI que le créateur trouve la force de sa création. Comme Dieu créant le monde DE RIEN, il invente, par sa grandeur INDIVIDUELLE, et donc différente de toute autre, des mondes neufs.

Lorsque l'analogie à l'acte de la Genèse devient caduque, que l'athéisme apporte sa sombre fatalité sur un monde abandonné, la création trouve alors chez l'homme la seule analogie restante : la maternité. Avec Rousseau, le poète devient la MÈRE de son oeuvre, alors qu'avec Corneille par exemple il en était bel et bien le PÈRE. Cette entrée de la maternité dans la théorisation de la création a pour conséquence de permettre une immédiate revendication créatrice des femmes. Cette période de l'histoire de la création sera alors celle d'une LUTTE sauvage 
pour réduire, par la création, la femme à sa place d'unique procréatrice castratrice et charnelle. Bien peu de femmes surnageront dans le carnage artistique du 19 e siècle et il importe de s'interroger, dès à présent, sur le regard rétrospectif que l'on porte sur elles.

Mais revenons à ce que l'on peut appeler la théorisation SEXUALISÉE de la création romantique et post-romantique. Dans son étrange et permanente RIVALITÉ créatrice, le créateur affirme alors, par analogie à la mise au monde, son autonomie souveraine. Il est le système producteur autonome de l'oeuvre. Lui, met au monde, comme une mère, dans la souffrance et l'AMOUR une oeuvre unique (au sens de UN), parce qu'il le fait seul, quand une fois encore, la femme a besoin de l'autre pour procréer. Plus d'analogie avec Dieu, mais une divinisation du créateur. Il est et reste l'UN, c'est-à-dire DIEU, la femme étant alors définitivement l'autre.

On comprend immédiatement ce qu'une telle théorisation - qui est encore celle de la création masculine actuelle - a de capital dans le thème de l'intimité. Venant de intimus, superlatif correspondant au comparatif interior, l'intimité implique le summum de l'intériorité, ce que l'être puise au fond de soi dans l'UNICITÉ de sa force vitale. Ce thème relie d'emblée l'intimité, l'unicité, et l'ORIGINALITÉ, forme majeure du retour à l'ESSENCE comme fondement de l'expérience créatrice. Or nous savons parfaitement que l'une des batailles les plus dures de la création féminine consiste à prétendre à l'originalité qui lui est déniée historiquement par la critique masculine. On pense aux durs propos de Baudelaire concernant celle qu'il appelait la femme $S A N D$. On pense à ce que récemment encore on nommait les ouvrages de dames. On pense par opposition à cela, chez Picasso, même dans l'univers du portrait, au refus d'utiliser le modèle, c'est-à-dire quelqu'un d'extérieur à soi, pour privilégier le rapport à soi, le rapport totalement intime qu'il a face à ce modèle, afin d'exprimer sa perception intérieure de l'être qui lui fait face et qu'alors il recrée totalement par la force de son retournement sur soi, par la volonté d'exprimer l'essentiel de son moi intime.

Or ce thème de l'originalité ou du retour à l'essence a été - est encore - la pierre d'achoppement de la création féminine telle qu' elle est vue et regardée par l'autre sexe. Quand on songe que la vision issue de Rousseau de l'intelligence féminine est qu'elle est destinée à son UTILITÉ propre, c'est-à-dire à lui permettre de PLAIRE à l'homme dont elle dépend (voir $L^{\prime} E$ Émile, livre V), on se pose la question de la pertinence de l'attente critique des femmes artistes et créatrices par rapport à l'autre 
sexe. La notion de PLAIRE d'abord, indique tout de suite celle de la dépendance au jugement et au désir de l'autre. Or, on sait combien l'artiste androgyne, c'est-à-dire celui qui s'affirme la mère autonome de son oeuvre, refuse le jugement social. Par opposition à cette nécessité du plaire, il affiche la force de l'expression de son intériorité unique et originale. Bien plus : ne pas plaire, c'est être, c'est affirmer cette originalité qui fait que l'on est sans modèle et sans rival. On sent souvent chez les créatrices cette angoisse d'être dans le paradoxe du plaire pour être (comme une femme), et ne pas plaire pour être (comme un homme). Pour en sortir, les femmes doivent CONCEVOIR LEUR PROPRE théorisation créatrice.

Car il me semble capital de comprendre que la nécessité affirmée del'intimité est une nécessité affichée par une rivalité théorique avec la création féminine. L'homme s'affirme créateur AUTONOME ET SOLITAIRE parce qu'il met en évidence cette création comme une non-procréation, comme une opposition paradoxale avec la MATERNITÉ. La nécessité de l'intériorité comme retour à l'essence n'a d'autre exigence que de confondre la femme en affirmant, dans la littérature masculine, dans l'art masculin, que la femme est dépourvue d'essence, que c'est un être qui pour être - et parce qu'elle est dépendante - doit être ÉDUQUÉ. Cette conception postrousseauiste de l'homme porteur d'un sens inné, essentiel, du BIEN (et par extension sans doute du BEAU) a d'ailleurs pour conséquence tragique une bénévolence à l'égard des pulsions dites fondamentales des hommes. Ainsi, on peut dire qu'on n'éduque pas les hommes : autrement, il y a longtemps qu'on cesserait de s'attendrir sur leurs prétendues pulsions sexuelles naturelles qui font de certains d'entre eux des violeurs, des pères incestueux. On ne leur apprend pas que ces pulsions ne sont pas légitimes. Bien plus, des sociologues, des psychologues, des psychiatres viennent de nous dire que tout cela est le fait d'une NATURE masculine qui les dépasse. Mais cette nature, on ne leur apprend pas qu'elle les pousse à des actes illégitimes. Et cela, parce qu'une vision particulière fait des hommes les détenteurs uniques d'une puissance fondamentale que, par opposition à ce que Rousseau a appelé l'absence de nature des femmes, on a voulu comprendre et préserver.

Ceci ne nous emmène pas si loin de notre sujet. Ce qui a pu faire la sublimité d'une pulsion vers le Beau, est fondamentalement poussé vers la rivalité avec le même objet : les femmes. 
L'intimité, c'est aussi le rapport de soi dans la connaissance de soi. Connaissance de soi qui est l'apanage du créateur romantique (on pense à Baudelaire, après Rousseau) qui a l'intuition de l'être et du monde. Cette perception immédiate du monde fait aussi du créateur celui qui entretient avec l'autre homme une intimité symbolique et fraternelle: ( $O$ homme qui crois que je ne suis pas toi). Qui lui donne aussi la connaissance de la femme, une connaissance qu'il tient de sa nature féminine d'androgyne. La femme est alors cernée, réduite à un objet de connaissance, partie féminine du créateur androgyne qui l'attire comme un sujet ou un modèle permanent. Sujet qui, dans la littérature, est presque toujours sacrifié. Sacrifiée Madame Bovary, sacrifiée Anna Karénine, sacrifiée Madame de Rénal, sacrifiée Madame de Mortsauf, etc. Sacrifiée aussi la partie féminine du créateur, cette vision passive, et évanescente, maladive et charnelle de lui-même, qui l'empêche d'atteindre à l'héroïsme viril. Et c'est Julien Sorel qui décide de se punir par la mort d'avoir cédé à un entraînement passionnel, c'est-à-dire à ce qu'il voit comme une pulsion du féminin en lui.

Or le rapport à soi est l'un des thèmes majeurs de la création masculine. Descente en soi qui est l'expérience symbolique de Dante, mais aussi le regard permanent sur soi qui est le fondement de la recherche Baudelairienne du dandy qui s'exprime dans la phrase célèbre : Être un grand homme et un héros pour soi-même. C'est que dans le thème del'intimité créatrice, le social disparaît. L'autre - et notamment l'autre sexe - est celui ou celle qui gêne l'expression de la solitude créatrice, qui se met en travers du nécessaire rapport à soi qui est une recherche de soi, comme celle de Proust et de La recherche $d u$ temps perdu. Ainsi, Wenceslas Steinbock, le génial créateur de la Cousine Bette de Balzac est constamment entravé par sa femme, personnage castrateur qui veut partager avec lui son souffle inspiré. Mais l'intimité ne se partage pas. Steinbock paye cette erreur de la perte de son inspiration créatrice. Il n'y a que de l'un dans la création masculine.

En 1947, dans un livre puissant: L'Invitée, Simone de Beauvoir tentera de résoudre cette difficulté de la création qui, selon le motto masculin, ne peut être que solitaire et retournement sur soi narcissique. Mais son personnage - qui est elle-même bien sûr - entretient avec un homme de théâtre, Pierre (qui n'est autre que Jean-Paul Sartre) une relation amoureuse et admirative d'une grande proximité. Françoise, l'héroïne, sait qu'elle a en elle une réelle puissance créatrice. Elle sait aussi qu'elle ne peut l'exercer - du moins le croit-elle - que sans Pierre. Alors, elle 
trouve un stratagème dialectique qui va tenter bien des femmes : prétendre qu'elle et lui ne font qu' un, affirmer que L'UN EST L'AUTRE. Ainsi, le tour serait joué. Ils seraient deux et cependant UN. Ils ne seraient pas, ensemble, une entrave à la création de chacun. Malheureusement, l'expérience ne marchera pas, à cause de l'invitée, qui fera passer l'un et l'autre à une troisième. Françoise devra se débarrasser de cette troisième.

Tout le combat de la femme pour son droit à la création s'exprime dans ce livre étonnant mais cependant insatisfaisant puisqu'il essaie de convertir, à l'avantage de la femme, pour son bénéfice, une théorisation où elle doit être sacrifiée. Pour réussir cet effort, elle doit s'identifier totalement à l'homme, Pierre, et sacrifier son identité de femme, ce qu'elle fait facilement puisqu'elle considère- et elle le démontrera dans Le deuxième sexe-qu'en ce qu'elle est femme, la femme est aliénée (On ne naît pas femme on le devient).

Or, toute la difficulté créatrice des femmes dans ce schéma de l'androgyne, tient précisément à cette nécessité de trouver en soi, à l'intérieur de soi, dans son intimité absolue, la source de sa création, alors que toute la création masculine et particulièrement la littérature, lui démontre qu'elle n'a pas d'en soi, qu'elle ne peut atteindre à l'UN, qu'elle est l'autre et donc entrave, poids, et pure procréation dépendante. Ainsi donc, pour atteindre l'intime, la femme doit-elle renoncer à la maternité? Problème essentiel de toute forme de création qui est pour la femme un problème ontologique que la théorisation masculine lui fait injustement assumer.

Car, ce qui est étrange, c'est que l'intime est, par un autre sens dérivé du premier, complètement lié au féminin. L'intime, au sens du privé, est le lieu privilégié où la théorisation masculine a historiquement confiné les femmes. En France, les deux magazines les plus traditionnellement féminins, les plus populaires, s'appellent Intimité et Nous deux. C'est que la sphère privée est celle où s'exprime l'affectif, que la théorisation masculine abandonne aux femmes. L'affectif, l'amoureux, c'est $l^{\prime}$ univers où l'AUTRE prime sur l'UN, où l'autre est plus important que $l^{\prime} U N$. Face à l'égoïsme narcissique de la création masculine, l'affect est l'apanage traditionnel des femmes. Et l'on peut se demander si une vision nouvelle de la création ne serait pas d'y faire vivre plus d'affect, plus d'autre, plus d'altruisme. Ainsi, un film récent qui a eu un prix au Festival de Cannes et qui raconte l'histoire de la recherche de sa mère par une jeune femme noire qui découvre alors que cette mère biologique est 
blanche. Secrets et mensonges est pour moi l'expression d'un système créateur nouveau où la recherche égoïste de soi cède le pas à l'affectif, aux rapports altruistes, et j'ai personnellement trouvéle film magnifique et réconfortant.

Que les créatrices aient le droit à l'intime, c'est-à-dire à l'expression introspective de leur intériorité essentielle et originale, cela ne fait aucun doute. Mais pour moi, la notion doit être débarrassée de toute sa violence anti-féministe. Cette intimité, qui est autonomie souveraine pour les hommes en tant qu'ils ne mettent pas d'enfant au monde, une autonomie donc qui s'oppose depuis toujours à la maternité, est une notion affreusement castratrice pour les femmes et certainement source d'angoisse créatrice incessante. L'en soi n'a nul besoin de s'exprimer contre l'autre et l'une ne saurait éliminer l'AUTRE. Ce que j'ai essayé de montrer - trop brièvement, cela est vrai - c'est que nos concepts sont piégés. Ce qu'il y a d'admirable dans la création doit être repensé par nous, les femmes, en le débarrassant de ce qui nous sacrifie. Autrement, le prix intime à payer est trop lourd.

Une fois posée l'aptitude indiscutable des femmes à la création, lorsqu'elles sont libérées de la théorisation masculine qui leur interdit précisément le récit d'une intimité par manque d'essence, par déficience naturelle, il est clair que la création, comme expression de la remontée de l'intériorité de l'être, comme mise à jour de l'identité, c'est-à-dire de $l^{\prime} U N$, est le summum de la qualité de l'être. La question qui se pose alors reste celle de la traversée du social, qui implique, pour les femmes, de DÉSAPPRENDRE, ce qui a pu faire dire à Simone de Beauvoir qu'on ne naît pas femme, mais qu'on le devient. L'effort créateur est pour les femmes un effort double. Celui de la descente en soi, pour puiser ce qui est essentiel, celui de l'oubli ou de la remise en question, c'est-à-dire de la critique de ce que la société leur apprend de soi. Si bien qu'il est difficile de concevoir qu'ily ait pour les femmes un récit intérieur, qui ne soit également la formulation d'une réelle critique de ce qu' on leur a imposé d'être, une expression souvent marquée par l'ironie, la provocation, ou le refus. Ce phénomène de critique du social n'est pas le même que celui que doivent ou peuvent concevoir les hommes, confortés quant à eux dans leur nature, leur identité, leur légitimité créatrice, par une puissante et historique théorisation. Certes, le créateur mâle se voit souvent comme un anti-social parce que son effort de descente en soi, son voyage orphique, en font l'être seul et donc opposé — parce que dominant aux organisations collectives. Mais la société, tout en la rejetant parfois, 
l'admire et lui donne la notoriété dont il a besoin, la visibilité qu'il recherche, même si cette visibilité est une critique ou un rejet apparent. Pour les femmes, tout se passe comme si cette intimité de soi, avec soi, exprimée par l'art, ne trouvait pas le regard intéressé de l'autre. Tout se passe trop souvent comme si le voyage en soi ne trouvait pas l'écho de l'universalité reconnue. C'est que la société trouve encore en la femme créatrice l'expression de la SPÉCIFICITÉ face à l'universalité masculine. Récit d'une intimité certes mais trop souvent limité au privé pour le regard social. Ainsi, Michel del Castillo qui raconte interminablement dans ses romans sa saga familiale, est-il reconnu comme un grand écrivain exprimant la hantise du rapport absolu à la mère, quand Annie Ernaux, admirable écrivain, épurée, se fait critiquer pour l'utilisation exclusive qu'elle fait de sa biographie, que certains jugent futile, sale ou inintéressante. C'est qu'Annie Ernaux, dans sa volonté d'expression de l'intime, va jusqu'aux limites de la négation du verbe, verbe historiquement masculin, porteur de toute l'aliénation à quoi je faisais allusion précédemment. Elle limite son récit au strict minimum matériel, refusant d'utiliser les moyens, et les techniques de la création masculine: le lyrisme, l'accumulation verbale, le beau style, l'allitération, etc. Elle se veut sans matière, plongeant dans son histoire avec un dépouillement d'entomologiste pour en restituer l'essence, la pure essence, l'intime absolu. Et il est clair que dans cette recherche créatrice, le problème de la MATIÈRE, du matériel, des MATÉRIAUX utilisés ne saurait être éludé, car il se pose, pour les femmes, par rapport à l'essence ou à l'intime, dans des termes différents de ceux des hommes. Trop longtemps confinées à la matière, elles cherchent à la réduire ou l'accumulent par provocation sans que jamais les moyens de l'expression-que ce soit en art, que ce soit en littérature - ne soient qu'un problème d'esthétisme. L'esthétisme, aussi, est piégé pour les femmes.

En conclusion, on peut dire que le retour à l'essence, la force du je dans le récit, l'expression de la vie intime de l'être doivent certes être les puissants leviers de la création féminine, mais que le combat à mener pour la reconnaissance de cette force créatrice est encore à gagner. Trop de questions se posent, dans la création, sur l'identité féminine, avec ses pulsions vers l'autre, sa recherche del'affect, face à l'identité masculine, définie par les hommes comme un narcissisme solitaire, égoïste et dominateur, pour que l'universalité puisse englober encore le double travail poétique. Et cependant, il est clair que c'est dans le dépassement du social que peut s'exprimer une légitimité équitable entre les deux créations. 